\title{
Théorie statistique des débits d'étiage ${ }^{(1)}$
}

\section{The statistical theory of low water discharges}

\author{
PAR E.-J. GUMBEL, \\ PROFESSEUR A COLUMRIA UNIVERSITY, NEW YORK (2)
}

Communication présentée lors de la réunion partielle de la Société Hydrotechnique de France (Section d'Hydrologie statistique. - Commission pour l'étude des débits de crue) organisée à Paris le 12 septembre 1957.

\begin{abstract}
Le débit d'étiage est defini en analogie avec le débit de crue comme le plas petit parmi les 365 débits jonrnaliers de l'année. C'est donc une valeur eatrême non négative. Dans l'ignorance de la distribution initiale des débits journaliers, on choisit pour l'analyse la troisieme distribution asymptotique de la plus petite valeur, distribution qui possède une limite inférieure $\mathrm{a}$ qui pourratit être nulle. En plus, deux autres parametres, le débit d'étiage caracléristique u et un facteur scalaire $\mathrm{k}$ existent dans cette distribution. Par définition, le débit d'étiage diminue si la durée de retonr augmente.

Si la limite inférienre est nulle, l'emploi da papier à probabilité extrémale conduit à une ligne droite pont le logarithme des débits d'étiage $\mathrm{x}$ en fonction d'une variable réduite $\mathrm{y}$. Si la limite inférienre est positive, on utilise la méthode des moments pour l'estimation des trois parametres.

Mais cette méthode ne garantit pas que l'estimation de la limite inferieure soit moindre que le plus petit débit d'étiage observé. Pour assurex cette propriété, on identifie cette observation avec la médiane de la plas petite valenr pour $Y$ observations. En outre, on utilise la moyenne arithmétique et l'écart lype pour l'estimation des denx aulves parametres.

La representation des débits d'étage ainsi obtenue peut servir à la prévision nécessair. pour la construction des réservoirs d'ean et l'irrigation des territoires secs, pourvu que le nombre d'observations soit suffisamment grand el ponrou que le climat ne change pas sensiblement dans l'époque à prévoir.
\end{abstract}

(1) M. le Professeur Gumbel a traité, le 23 avril 1956, devant la Société Hydrotechnique de France, la théorie tos valeurs extrêmes appliquée aux débits maxima de crue. Cet exposé avait été suivi d'une discussion au cours de laquelle $M$. Bernier avait, dans une importante intervention, comparé la loi de Gumbel à diverses autres lois (Galton-Gibrat, Fréchet) dans leurs applications à l'évaluation des débits maxima de crues. Ces exposés

\begin{abstract}
Low water discharge is defined analogously with flood discharges as being the lowest of the 365 daily discharges during the year Not knowing the initial daily discharge distribution, the third asymptotic distribution of the smallest value is selected for purposes of analysis. This distribution has a lower limit which can be zero. In addition, this distribution has two other parameters, the characteristic low water discharge u and a scalar factor k. By definition, the low water discharge becomes smaller if the return period increcses. If the lower limit is zero, the use of extremal probability paper results in a straight line for the logarithm of low water discharges $\mathrm{x}$ as a function of a reduced variable $\mathrm{y}$. If the lower limit is positive, the three parameters are estimated by the method of moments.

But this method does not guarantee that the estimated lower limit will be smaller than the smallest observed low water discharge. To make sure that this is true, this observed value is identified with the median of the smallest value for $N$ observations. Moreover, the arithmetic mean and the standard deviation are used to estimate the other two parameters.

The representation of low water discharges obtained in this way, can be used for making forecasts in connection with the construction of resernoirs and irrigating dry regions, provided that the number of observations is large enough and provided the climate does not change appreciably during the period for which the forecast is made.
\end{abstract}

ont paru daus la Houille Blanche de $1956, \mathrm{n}^{\circ} 5$.

Au cours d'une seconde réunion de la S.H.F., le 12 septembre 1957 , M. Bernier présenta un nouvel exposé sur la comparaison des lois de Gumbel et de Fréchet (Cf. p. 47 du présent numéro), tandis que M. Gumbel consacrait aux débits d'étiage la communication ci-après qui constitue une application nouvelle de la théorie des valeurs extrêmes.

(2) Ecrit sous les auspices du Higgins Fund. 
Cet exposé concerne l'étude statistique des débits d'étiage et non celle de la sécheresse. La sécheresse est en effet un facteur météorologique vaguement défini, alors que le débit d'étiage est une notion hydrologique précise. Parmi les 365 débits moyens journaliers de l'année, il en est un ou plusieurs qui sont plus petits que tous les autres. C'est le débit d'étiage.

Il s'agit done ici de la théorie statistique des plus petites valeurs, analogue à celle des plus grandes valeurs appliquée aux crues.

L'étude des débits d'étiage présente un très grand intérêt pour la construction des réservoirs, dont la durée de remplissage est liée à la valeur du débit minimum. La connaissance de ce dernier a aussi un intérêt hygiénique; le débit doit ctre assez fort pour emporter les détritus que l'on jette dans le cour's d'eau. Il est donc de première importance de connaitre la variation du débit d'étiage au cours du temps.

On considère une variable aléatoire (débit) $\mathrm{X}$ positive. (Il n'existe pas de débit négatif.) Soit :

$$
\mathrm{P}(x)=\operatorname{Prob}[\mathrm{X} \geqq x]
$$

Cette probabilité décroît si $x$ croît. Si la distribution initiale des débits journaliers est connue et s'il y a indépendance, la forme $\mathrm{P}(\boldsymbol{x})$ pour le plus petit débit est connue. Dans l'ignorance de la distribution initiale, il est nécessaire d'employer la théorie asymptotique des plus petites valeurs.

\section{THEORIE ASYMPTOTIQUE DES PLUS PETITES VALEURS}

Il existe trois formes de distributions asymptotiques des plus petites valeurs valables pour un grand nombre d'observations :

I.

$$
\mathrm{P}(x)=\exp \left[-e^{\alpha(x-u)}\right] \quad \alpha>0
$$

Cette loi est obtenue dans le cas d'une distribution initiale du type exponentiel. Tous les moments existent. La variable $x$ est illimitée dans les deux directions.

$$
\text { II. } \quad \mathrm{P}(x)=\exp \cdot\left[\frac{-(\omega-u) /(\omega--x)}{u<\omega, x \leqq \omega, k>0}\right]^{7}
$$

Il existe donc ici une limite supérieure, mais pas de limite inférieure pour le débit. Par contre, les moments d'ordre supérieur n'existent pas.
Les deux formules ont le désavantage d'affecter des probabilités non négligeables à des valeurs négatives non existantes.

III.

$$
\mathrm{P}(x)=\exp _{u>\varepsilon, x \geqslant \varepsilon, k>0}[-(x-\varepsilon) /(u-\varepsilon)]^{*}
$$

Cette loi suppose l'existence d'une limite inférieure $\varepsilon$ qui pourrait être nulle. Tous les moments existent. Dans les trois formes, le paramètre $u$ est tel que : $\mathrm{P}(u)=1 / e$. Ce paramètre est appelé : débit d’étiage caractéristique.

Pour le choix d'une distribution asymptotique, on suppose l'existence des moments des distributions initiales des débits. Cela étant, on utilise alors la troisième forme qui, seule, donne des débits d'étiage positifs, à condition que : $\varepsilon \geqq 0$.

\section{DUREE DE RETOUR}

Considérons d'abord le débit d'étiage en fonction du temps. Soit la durée de retour :

$$
\mathrm{T}(x)=1 /[1-\mathrm{P}(x)]
$$

Si le débit d'étiage diminue, la durée de retour augmente. Avec la forme III choisie, et d'une façon asymptotique, à savoir des petites valeurs de $X$ qui nous intéressent :

$$
\log \mathrm{T}=+k[-\log (x-\varepsilon)+\log (u-\varepsilon)]
$$

et dans le cas où la limite inférieure est nulle :

$$
\log \mathrm{T}=+k[-\log x+\log u]
$$

Cette dernière formule, donnant le logarithme de la durée de retour comme fonction linéaire du logarithme du débit d'ćtage, est à opposer à la formule analogue pour les crues, qui donne $\log \mathrm{T}$ en fonction linéare do débit de crue. Il est intéressant, d'apros cotte fommule, de connaître, pour $\varepsilon=0$ :

$1^{\circ}$ le débit d'étiage o. qui double la durée de retour ' $\mathrm{T}$ :

$$
a_{m}, x_{1}, 1 / 21 / k
$$




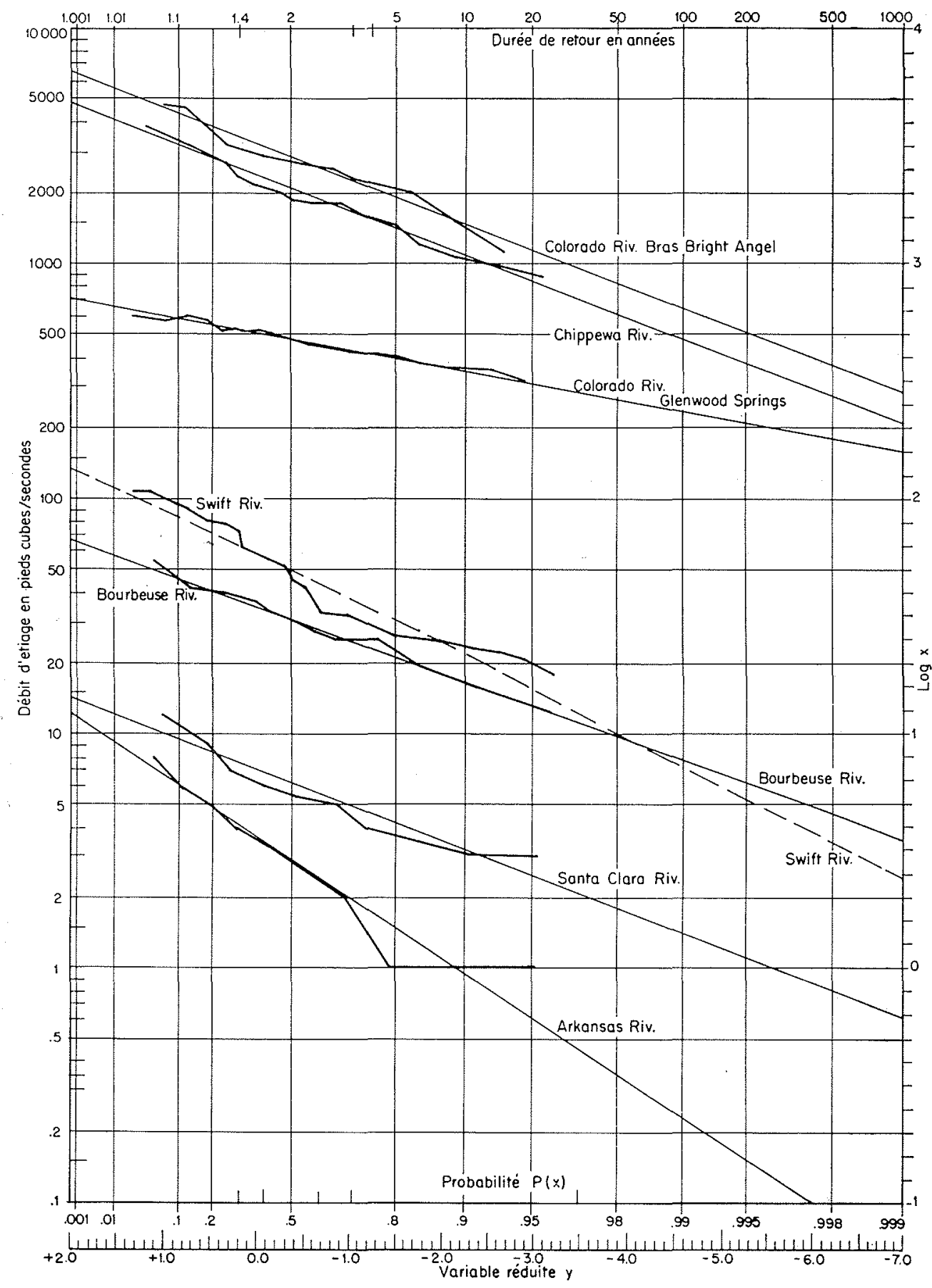

Frg. 1

$2^{\circ}$ la durée de retour pour un débit d'étiage de moitié inférieur à un débit donné :

$$
\mathrm{T}(x / 2)={ }^{\mathrm{T}}(x) 2^{k}
$$

Rappelons, à ce propos, la formule donnant la durée de retour d'une crue deux fois plus forte qu'une valeur donnée :

$$
\mathrm{T}(2 x)=\mathrm{T}^{2}(x) \cdot e^{x t u}
$$

La comparaison des deux formules montre la différence essentielle entre l'analyse des crues et celle des débits d'étiage. La durée de retour du double de la plus grande crue observée est de l'ordre du carré des nombres d'années observées, tandis que la durée de retour de la moitié du plus sévère débit d'étiage observé est seulement un multiple des nombres d'années observées. 


\section{MÉTHODES GRAPHIQUES}

Si l'on introduit dans la formule III une variable réduite $y$ définie par :

$$
\mathrm{P}(x)=\exp \left[-e^{y}\right]
$$

alors :

$$
y=\log [-\log \mathrm{P}(x)]
$$

On obtient ainsi une relation linéaire entre le logarithme du débit $x-\varepsilon$ et la variable réduite $y$, à savoir :

$$
\log (x-\varepsilon)=\log (u-\varepsilon)+y / k
$$

Partant des tables de la loi doublement exponentielle :

$$
\Phi(y)=\exp \left[-e^{-y}\right]
$$

publiées par le National Bureau of Standard [1], il est loisible de tracer un papier à probabilité pour les plus petites valeurs. Dans le papier à probabilité des plus grandes valeurs, on change $y$ en $-y$ et $\Phi$ en $\mathrm{I}-\mathrm{P}$.

On porte (voir fig. 1) en ordonnée : le logarithme du débit, et en abscisse la variable $y$ en échelie linéaire, mais décroissante de gauche à droite; alors on obtient facilement deux autres échelles, l'une graduée en probabilité, l'autre en durée de retour.

Pour tracer la courbe expérimentale, on prend les $\mathrm{N}$ débits d'étiage en ordre décroissant (en partant du plus grand vers le plus petit) et on affecte à la $i^{\text {ème }}$ valeur la fréquence empirique : $i /(\mathrm{N}+1)$.

Si $\varepsilon=0$, on obtient la droite $\log x=\log u+y / k$. qui peut être extrapolée en fonction des durées de retour.

Les graphiques 1 à 5 ont été tracés de cette façon. Bien que le nombre des observations ne soit pas très grand, il est intéressant de noter la décroissance du débit d'étiage en fonction du

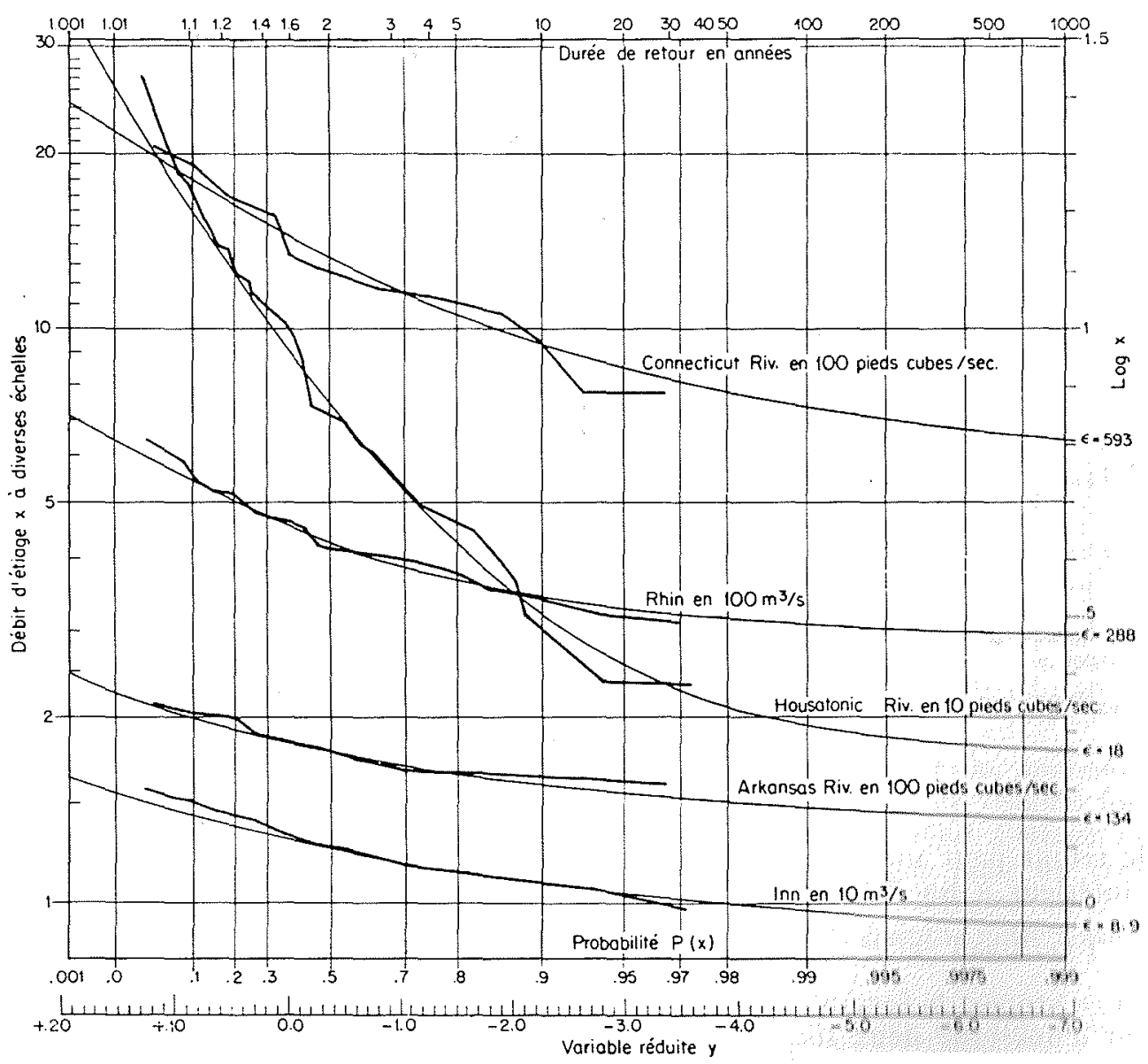

I'II, 2 
temps. Pour certaines rivières, la décroissance est lente, le danger est minime. Pour d'autres cours d'eau, la décroissance est plus rapide, le danger est grand.

Même si on n'accepte pas la théorie statistique, cette méthode a le mérite de fournir une manière rationnelle de tracer les débits d'étiage en fonction du temps. On peut accepter cette théorie, pourvu qu'elle représente bien les observations. Il semble que ce soit le cas dans les exemples traités.

Pour réduire différentes rivières à une même échelle, on pourrait diviser le débit d'étiage par la grandeur du bassin versant.

\section{ESTIMATION DES PARAMĖTRES}

L'estimation des paramètres dans le cas où $\varepsilon=0$ est le même que pour les débits de crue. Ón n'a qu'à remplacer $x$ par $\log x$ (voir fig. 1 ).

L'existence d'une limite inférieure introduit un troisième paramètre supplémentaire qui complique le problème d'estimation. Plusieur's méthodes peuvent ètre utilisées. Mais la méthode moderne, dite « maximum likelihood», n'est pas applicable.

Notre problème est de trouver un ajustement de la formule :

$$
\log (x-\varepsilon)=\log (u-\varepsilon)+y / k
$$

a) En premier lieu, UNE MÉTHOdE INTUITIVE, qui plaira aux ingénieurs, mais plaira moins aux statisticiens. On part d'un $\varepsilon$ quelconque et on trace la courbe. Suivant la concavité de cette courbe, on prendra un $\varepsilon$ plus grand ou plus petit jusqu'à ce qu'on obtienne une ligne de courbure opposée. On procédera ensuite par tâtonnements pour trouver un $\varepsilon$ intermédiaire qui donne une droite. Cette méthode évite les calculs et permettra l'extrapolation pourvu que l'ajustement de la droite soit bon. Au besoin, on pourrait estimer les paramètres $u$ et $k$ en remplaçant $\log x$ par les $\log (x-\widehat{\varepsilon})$, ce qui permet l'application de la méthode linéaire usuelle pour les crues.

\section{b) MÉTHOde DES MOMENTS :}

Etudions d'abord les valeurs caractéristiques de la distribution. On voit aisément que le mode et la médiane, ainsi que leurs distances, dépendent du paramètre $k$. En effet, le mode $\tilde{x}$ et la médiane $x$ sont les solutions de :

$$
\begin{aligned}
& \check{x}-\varepsilon=(u-\varepsilon)(1-1 / k)^{1 / k} \\
& x-\varepsilon=(u-\varepsilon)(l y 2)^{1 / k}
\end{aligned}
$$

On en tire la relation : mode $\leq / \equiv$ mediane, si $1 / k \equiv / \equiv 0,30685$.

Cette distribution est donc plus souple que la distribution des crues, où le mode est fixe et identique à la valeur caractéristique $u$.

De façon générale, on utilisera la méthode des moments pour estimer les trois paramètres. Le moment d'ordre $l$ est :

$$
[(x-\varepsilon) /(u-\varepsilon)]^{l}=\mathrm{\Gamma}[\mathrm{I}+(l / k)]
$$

Avec cette formule, il est aisé de voir que le coefficient d'assymétrie de Pearson $\overline{\sqrt{\beta}}_{1}$ est fonction de $k$ seulement. Connaissant le $\overline{\sqrt{b_{1}}}$ observé, on peut alors déterminer $k$ par l'intermédiaire d'une table donnant $\sqrt{\beta_{1}(\kappa)}$. Ceci posé, on peut déterminer $u$ et $\varepsilon$ en fonction de la moyenne $\bar{x}$, de l'écart-type $s$, et de $k$, d'après:

$$
\begin{aligned}
& \widehat{u}=\bar{x}+s \mathrm{~A}(\widehat{k}) \\
& \hat{\varepsilon}=\widehat{u}-s \mathrm{~B}(\widehat{k})
\end{aligned}
$$

La référence [2] contient des tables donnant les fonctions $\sqrt{\beta_{1}(k)}$ et :

$$
\begin{aligned}
& \mathrm{B}(k)=\left[\Gamma(1+2 / k)-\Gamma^{2}(1+1 / k)\right]^{-1 / 2} \\
& \mathrm{~A}(k)=\mathrm{B}(k)[1-\mathrm{T}(1+1 / k)]
\end{aligned}
$$

La figure 2 illustre des ajustements effectués de cette façon.

Exemples :

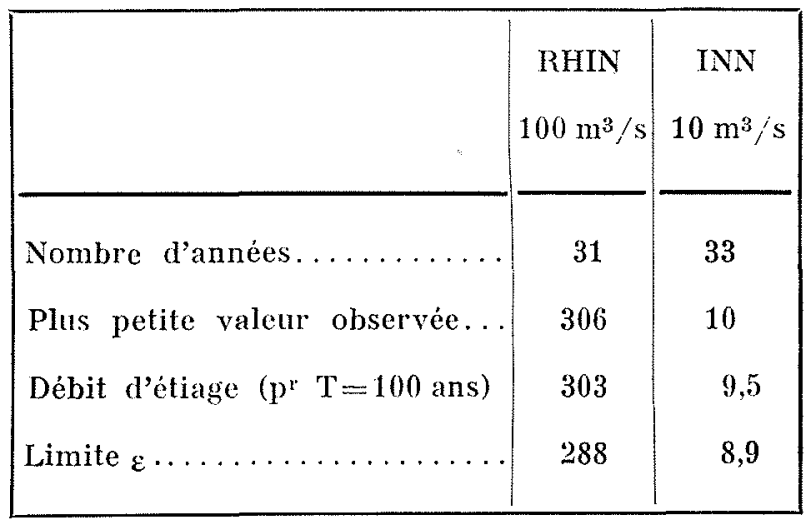

Il faut souligner ici qu'étant donné le petit compte des observations, des erreurs d'échantillonnage sont à craindre, surtout sur $\varepsilon$. L'étude de ces erreurs est à faire. Le problème ne sem- 
ble pas difficile si les paramètres sont estimés par la méthode des moments. Un autre problème à étudier est la détermination des courbes de contrôle.

\section{c) AttTre MÉTHOde d'estimation:}

Un autre procédé d'estimation repose sur la relation :

$$
\mathrm{P}(u)=1 / e \text {. }
$$

Il est loisible de déterminer $u$ par interpolation entre deux valeurs consécutives:

$$
\mathrm{P}\left(x_{m}\right)>1 / e \text { et } \mathrm{P}\left(x_{m+1}\right)<1 / e
$$

Il est cependant malaisé de combiner cette méthode avec l'estimation des autres paramètres. En effet, il est généralement souhaitable de conserver la moyenne et l'écart-type, ce qui conduit à l'estimation de la quantité : $(u-\bar{x}) / s$ qui est la fonction de $\mathrm{A}(\boldsymbol{k})$. Par malheur, la différence : $u-\bar{x}$ est très petite et les erreurs d'estimation sur $u$ et $\bar{x}$ amènent de grandes erreurs relatives sur la différence, ce qui détruit toute précision.

La méthode des moments ne garantit pas que la valeur de $\hat{\varepsilon}$ estimée soit inférieure au plus petit débit d'étiage observé $x_{1}$. Pour éliminer cette difficulté, nous proposons une méthode qui a un intérêt intuitif. Le plus petit débit d'étiage est l'observation la plus importante. Nous allons l'utiliser pour l'estimation des paramètres en introduisant l'hypothèse que la valeur observée est la médiane du plus petit débit d'étiage pour $N$ années.
Or, d'après la stabilité de la loi asymptotique des petites valeurs, on connait la loi du plus petit débit d'étiage. C'est une loi de même forme que la loi des débits d'étiage eux-mêmes; seul le paramètre d'échelle est multiplié par un facteur dépendant de $\mathbf{N}$. On utilisera done la médiane du plus petit débit d'étiage, $\widetilde{x}_{1}$ pour $\mathrm{N}$ années, la moyenne arithmétique $\bar{x}$ et l'écart-type $s$. La combinaison de ces trois valeurs conduit à :

ò̀ :

$$
\left(\bar{x}-\breve{x}_{1}\right) / \sigma=\mathrm{D}\left(\mathrm{N}^{\prime}, k\right)
$$

$\mathrm{D}(\mathrm{N}, k)=\left[\mathrm{\Gamma}(1+1 / k)-(\log .2 / \mathrm{N})^{1 / k}\right] \mathrm{B}(k)$

En traçant un tableau de $\mathrm{D}(\mathrm{N}, k)$, on obtient une estimation de $k$ si l'on remplace $\breve{x}_{1}$ par le plus petit débit d'étiage $x_{1}$ et $\bar{x}$ et $\sigma$ par la moyenne et l'écart-type $s$ observé. Enfin les deux équations mentionnées sous (b) donnent de nouvelles estimations du débit d'étiage caractéristique $u$ et du minimum $s$. Cette méthode garantit que l'estimation de a sera plus petite que la plus petite valeur observée.

Elle a été utilisée pour les débits d'étiage du fleuve Waikoto au lac Taïpo (Nouvelle-Zélande), 1906-1941. La moyenne et l'écart-type sont : $\bar{x}=3.425,3 ; s=537,2$ pieds cubes par seconde. Le plus petit débit d'étiage $x_{1}=2.380$ conduit à $\mathrm{D}(36, \widehat{k})=1,9456$, dont on tire le paramètre $1 / k=0,32475$, le débit d'étiage caractéristique $\widehat{u}=3.605$ et la limite $\varepsilon=1.912$. L'accord entre la théorie et les observations tracées dans la figure 3 ne laisse rien à désirer.

Débits d'étiage du Waikoto (Nouvelle-Zélande)

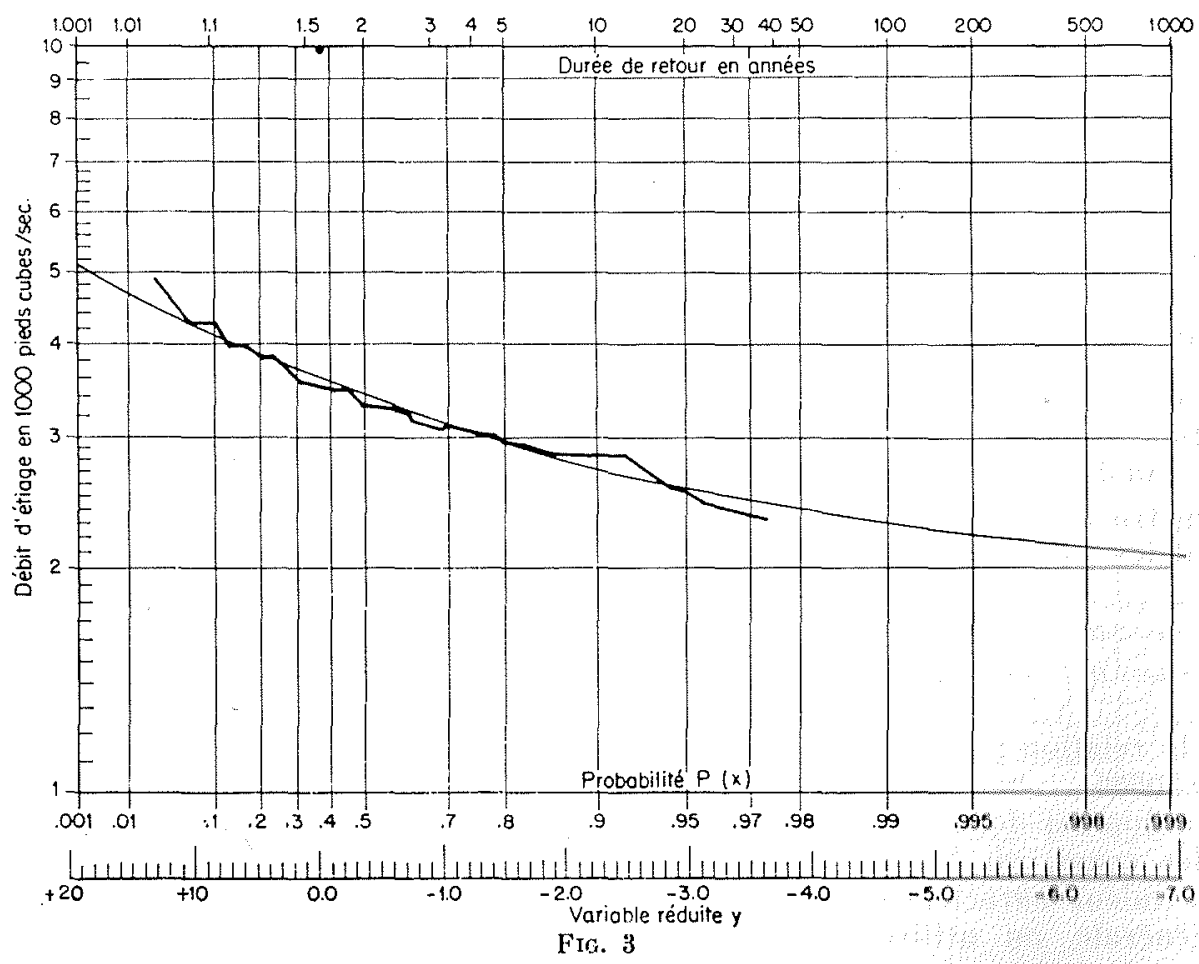




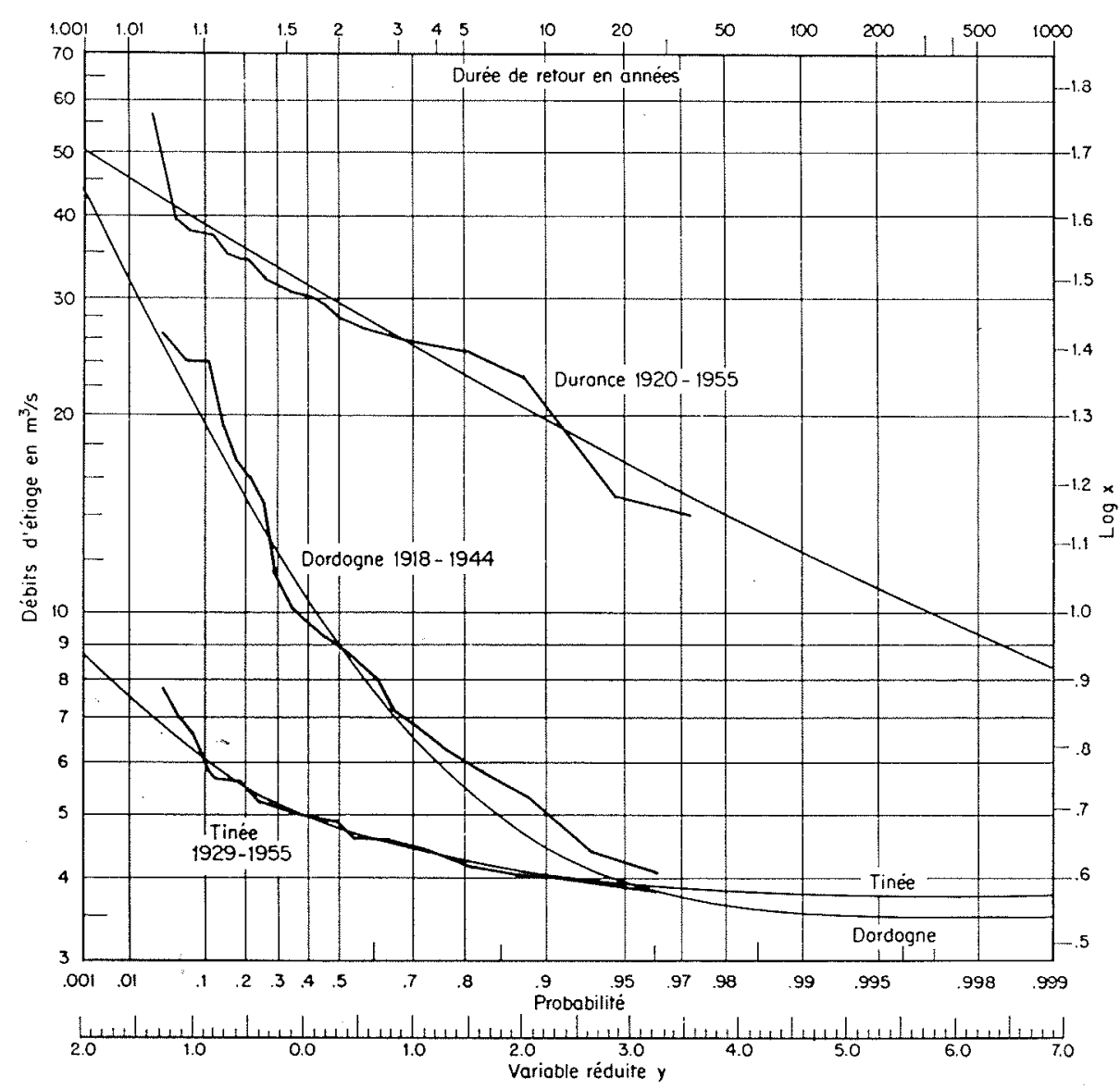

Fig. 4

Débits d'étiage du Rhin à Rheinfelden.

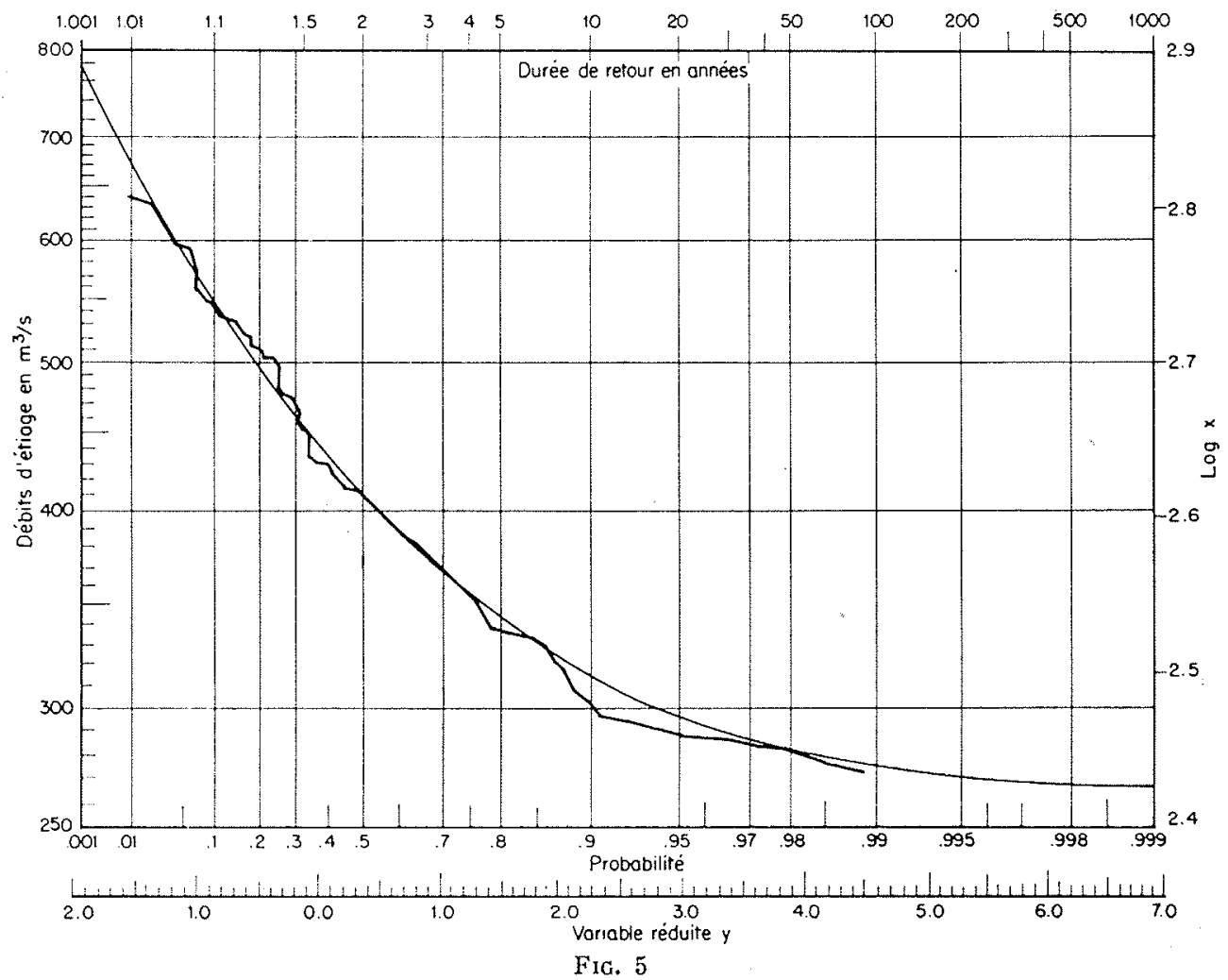




\begin{tabular}{|c|c|c|c|c|}
\hline \multicolumn{5}{|c|}{ DÉBITS D'ÉTI'AGE } \\
\hline Rivière . . . . & TINÉE & DORDOGNE & Dunance & RHin \\
\hline Lieu . . . . & Bancairon & Argentat & Ventavon & Rlyeinfelden \\
\hline Date $\ldots \ldots \ldots \ldots \ldots \ldots \ldots \ldots$ & $1929-55$ & $1918-44$ & $1920-55$ & $1869-1956$ \\
\hline Nombre d'années............ $N$ & 27 & 27 & 36 & 88 \\
\hline Moyenne ............. $\bar{x}$ & 4,893 & 10,952 & 29,388 & 420,67 \\
\hline Ecart-type $\ldots \ldots \ldots \ldots \ldots \ldots s$ & 0,846 & 6,316 & 7,311 & 89,73 \\
\hline Minimum $\ldots \ldots \ldots \ldots \ldots x_{1}$ & 3,800 & 4,000 & 14,000 & 272,00 \\
\hline Facteur $\ldots \ldots \ldots \ldots \ldots \ldots \ldots \ldots \ldots \ldots \ldots$ & 1,292 & 1,101 & 2,105 & 1,657 \\
\hline Paramètre $\ldots \ldots \ldots \ldots \ldots \ldots \ldots 1 / k$ & 0,700 & 0,867 & 0,250 & 0,533 \\
\hline Etiage caractéristique.......... & 5,01 & 11,05 & 32,08 & 441,2 \\
\hline Limite . . . . . . . . . . . & 1,31 & 3,40 & 3,33 & 258,2 \\
\hline
\end{tabular}

Le tableau ci-dessus contient les données numériques pour quelques rivières françaises. Les débits d'étiage observés et les valeurs théoriques calculées par la même méthode sont tracés dans les graphiques 4 et 5 .

Les deux estimations pour l'étiage caractéristique et la limite du Rhin obtenues par des méthodes différentes et basées sur 31 et 88 années, diffèrent par $3 \%$ et $10 \%$ respectivement.

Les graphiques indiquent clairement l'existence d'une limite inf'érieure pour le Rhin, la Tinée et la Dordogne. Pour la Durance, par contree, la question de savoir si la limite inférieure est positive ou nulle reste ouverte.
La théorie des valeurs extrêmes a eu un certain succès pour l'analyse des crues. L'expérience va nous montrer ce qu'elle vaut dans l'analyse des débits d'étiage.

\section{REFEERENCES}

[1] "Probability tables for analysis of extreme value data," National Bureau of Standard: Applied Mathematics, series 22 (1953).

[2] Gumbel (E.-J.). - "Statistical theory of droughts." Proceedings of American Society of Civil Engineers, vol. 80 , no. 439 . suivante :

Cette communication à la Société Hydrotechnique de France a domné lieu à la discussion

DIS C U S I O N

président: M. HuPnen

M. le Président souligne la nouveauté, pour un certain nombre d'auditeurs, du sujet traité par M. Gumbel et son intérêt, la sécheresse étant certainement une catastrophe, non seulement du point de vue sanitaire, comme l'a signalé le conférencier, mais aussi pour les Sociétés ou Organismes producteurs d'énergie hydraulique à faible rayon d'action et dont les réseaux électriques ne sont pas interconnectés. Quels que soient les pro- grès que puisse faire la thétio dontlavenir, M. Gumber aura eu le mérite d'avoir aborde lo promier ce problème.

M. le Président remarquo gav lo ou du débit absolument nul $(\varepsilon=0, x=0)$, conslitie $14 \pi$. Gumber comme jamais atteint parce qu'il a now pevode de retour infinie, est cependant susceptible do 16 promerter pour certaines rivières africaines pendant ploktowt mois do l'année; la

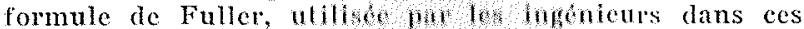


pays en cas de manque de données hydrologiques, montre que le débit fatal peut être nul au-dessous d'une cerlaine quantité de pluie tombée.

M. Gumbel répond qu'une rivière ne peut plus être considérée comme telle dès l'instant qu'elle ne coule plus. Il y a là des conditions climatiques et météorologiques qui ne sont pas prévues dans sa théorie.

M. le Président évoque aussi le eas de la rivière qui alimente une nappe ou de celle dont le débit décroît de l'amont vers l'aval, contrairement à ce qui se passe normalement, et demande si ce cas ne peut pas êtro considéré comme celui d'un débit négatif.

Divers exemples sont donnés de cas semblables, mais il ne semble pas que ces cas puissent être pris en compte dans une théorie seientifique appliquée à des phénomènes naturels, soit qu'ils appartiennent à la mythologie, soit qu'ils résultent d'interventions humaines sur Ies débits des rivières (pompages, dérivations, etc.), soit enfin qu'il s'agisse des circonstances naturelles tout à fait spéciales (mascaret, par exemple).

Cependant M. GIGnoux signale le cas concret d'unc retenue à usage hydro-électrique avec barrage étanche et sans soutirage, qui a vu son niveau baisser en étiage parce que, probablement, l'évaporation du lac artificiel dépassait les apports naturels (retenue du Viaur, à Thuries dans le bassin du Tarn, en 1938 ou 1939).

En réponse à une question de $M$. le Président, M. Gumbe précise que le débit d'étiage considéré est le plus faible des débits moyens journaliers observés dans l'année: son étude tend à déterminer la plus petite valeur probable de ce débit d'étiage.

La durée pendant laquelle a régné ce débit d'étiage n'intervient pas dans l'étude, car elle conduirait à un nouveau problème: distribution extrémale à deux variables, la valeur minimum et sa durée.

M. le Président souligne l'importance de la durée, dont les conséquences peuvent être relativement beaucoup plus graves pour un étiage que pour une crue.

Sur la demande de M. le Président, M. Magrin indique les rapports qui peuvent se présenter entre son travail sur la décroissance des débits de la Dordogne a partir d'un débit de crue, et la théorie de M. Gumber sur les étiages.

L'étude des débits de la Dordogne à Mavèges, rivière ¿ légime pluvial dominant, présentée à la S.H.F. en 1953 par M. MAGRIN, portait notamment sur la série chronologique des débits joumaliers en l'absence de précipitations.

Ces débits ont pu être ajustés à une courbe de décroissance exponentielle ayant pour asymptote:

- soit une valeur bien définie constante du débit (en

l'espèce $30 \mathrm{~m}^{3} / \mathrm{s}$ ) comparable ì la valeur « $\varepsilon$ plus grand que $0 \gg$ de M. Gramet. (C'est ce qui se produit après une période de hautes eaux, tant que le sol reste imprégné d'eau et il ne s’agit pas là de débit (l'étiage.)

soit en période d'étage, un débil nul comme daus lo cas envisagé par M. Gumbri, $\varepsilon=0$.

Le débit étant alors déterminé, si l'on connait te nombre de jours sans pluie compté à partir d'un débit donné $Q_{0}$, la plus petite valeur du débit d'étiage correspond à la plus grande valeur du nombre de jours sans pluie compté à partir de l'époque de $Q_{0}$.

La recherche de la plus petite valeur du débit d'étiage se ramènerait ainsi a la recherche de la plus grande valeur d'un nombre de jours sans pluie.

M. Gumber réfléchira à la réponse qu'il peut donner ì cette question, qui implique l'étendue des bassins ver- sants et la corrélation entre le débit et le nombre de jour's sans pluic.

Des questions sur les applications spéciales des travalux de M. Gumber sont posées au conférencier qui y répond très volontiers:

1" M. Nahon (Gaz de France) pense que la portée de la théorie des valeurs extrêmes dépasse le cadre des problèmes hydrologiques. En particulier, une question analogue se pose avec les émissions de gaz, liées à la température: la prévision des pointes d'émission impose en égard au développement du chauffage des locaux au gaz, d'étudier Ia probabilité d'apparition de très basses températures comme pour les débits de cours d'eau, les observations sont limitées en nombre). Certaines études ont été failes à ce sujet au Gaz de France, M. le Professeur Gumber a-t-il eu lui-même l'occasion d'appliquer la théorie des valeurs extrêmes à des problèmes autres qu'hydrologiques, en particulier aux températures?

M. Gumbel répond que la théorie des valeurs extrêmes est applicable a un certain nombre de variables qui, contrairement aux débits, peuvent descendre au-dessous de zéro: il en est ainsi des températures et aussi des pressions les plus basses qui, portées sur un graphique de probabilité extrémale à échelle linéaire, se rangent très sensiblement suivant une droite descendante (l'application aux températures est en effet utilisée par certaines usines à gaz aux Etats-Unis). La théorie de la plus grande valeur sapplique aussi aux vents les plus forts.

2" M. Danu (Société d'Etudes Pratiques de Recherche Opérationnelle) s'intéresse aux méthodes qui permettraient la prévision des hauteurs d'eau dans une rivière, ou plus exactement, du passage à un certain niveau (niveau minimum permettant encore la navigation).

M. Gumber répond qu'il s'agit d'un problème un peu différent: celui des distributions saisonnières. Il signale deux articles qu'il a écrits a ce sujet dans le «Journal de l'Association Américaine de Statistique»; l'un traite abstraitement d'une « distribution circulaire $\gg$ (Circular normal distribution), l'autre donne quelques applications pratiques. Ces articles ont été publiés dans le volume 48, pages 131 à 152 (1953) et dans le volume 49 , pp. 267 à 297 (1954).

Cette distribution circulaire normale est un bon moyen d'analyse de la distribution saisonnière et de prévision à l'échelle du mois ou de la semaine lorsqu'il y a un seul maximum et un seul minimum dans la période considérée et que ces valeur's extrêmes sont diamétralement opposées (d'une demie période).

Sur la demande de M. le president, M. Gumbri donne la référence de son mémoire:

Gumber (E.-J.). - Stastistical Theory of droughts (Proceedings A.S.C.E. - Hydratics division, mai 1954. Vol. 80, Séparate $\left.n^{\circ} 439.\right)$

Ce travail constitue la base de l'exposé de M. Gumber, auquel l'auteur a ajouté quelques références d'applications récentes de sa théorie à certaines rivières. Il propose de l'appliquer à des observations sur des rivières françaises qu'on voudra bien lui communiquer.

M. le Président remercie vivement M. Gumbel d'avoir exposé à la S.H.F. le problème, délicat et très important a de nombreux points de vue, de la sécheresse et lui demande de bien vouloir nous reserver un nouveau rendez-vous à l'occasion d'un de ses prochains passages à Paris, en vue d'une discussion plus approfondie de sa conférence de ce jour, aprés diffusion du compte rendu qui en sem fait. 